\title{
A disturbance beyond the barrier-chronic kidney disease allows angiotensinogen invasion
}

\author{
Masaki Mogi ${ }^{1}$
}

Received: 31 March 2021 / Revised: 9 April 2021 / Accepted: 13 April 2021 / Published online: 28 May 2021

(c) The Japanese Society of Hypertension 2021

Circadian rhythms are physical, mental, and behavioral changes within sleep/wake cycles and hormonal activity during the 24-hour day and are closely related to cardiovascular events [1]. Multiple biochemical and physiological parameters, such as blood pressure, autonomic nervous system activity, and renin-angiotensin system (RAS) activity, exhibit circadian rhythms. Circadian rhythms and renal function have been studied for more than 150 years [2], and there has been a focus on the association of circadian rhythm disorders of renal function with hypertension, chronic kidney disease (CKD) and renal fibrosis [3]. Ohashi et al. studied the relation between circadian rhythms and renal damage, especially focusing on the intrarenal RAS, using various rodent models of renal injury [4, 5]. They previously contributed a review article to Hypertension Research reporting that an increase in the intrarenal angiotensinogen (AGT) level induced by augmented glomerular permeability affects circadian rhythms of the intrarenal RAS [6]. Here, the present study by Matsuyama and Ohashi et al. demonstrated that intrarenal RAS activity is influenced by the filtration of "liver-derived" AGT from damaged glomeruli due to disordered circadian fluctuations of glomerular capillary pressure [7].

AGT in the kidney is produced by the proximal tubule [8]. As shown in previous studies, due to its molecular size [9], the glomerular permeability of AGT is limited; thus, the intrarenal RAS is independently regulated [10] (Fig. 1). However, Matsuyama et al. demonstrated here that liverderived AGT is filtered through the "damaged glomeruli" into the kidney and induces intrarenal RAS activation, resulting in blood pressure elevation, renal damage, and circadian fluctuations. They used rats with Adriamycininduced nephropathy, which causes severe proteinuria due

Masaki Mogi

mmogi@m.ehime-u.ac.jp

1 Department of Pharmacology, Ehime University, Graduate School of Medicine, Shitsukawa, Tohon, Ehime, Japan to podocyte injury without a reduction in the estimated glomerular filtration rate (eGFR), to avoid significant renal decline. Here, circadian changes in intrarenal AGT expression and the diameter ratio of afferent to efferent arterioles (A/E ratio) were observed in Adriamycin-injured rats although there was no significant change in systemic blood pressure. Moreover, treatment with an angiotensin II receptor blocker, olmesartan, prevented such changes, probably via suppression of efferent arteriole vasoconstriction. Therefore, AGT is thought to be increased in the awake state due to an increase in glomerular capillary pressure but not due to systemic blood pressure. Matsusaka et al. also reported that liver-derived AGT is the primary source of renal angiotensin II and that an abnormal increase in permeability of the glomerular capillary wall enhances renal angiotensin II production using kidney-specific angiotensinogen knockout mice [11]. These results indicate that liver-derived AGT may "invade" the kidney by filtration from damaged glomeruli and disturb the intrarenal RAS and circadian rhythms of the kidney, resulting in blood pressure elevation and enhancement of renal damage, while renal plasma flow, GFR and tubular reabsorption and/or secretion processes physiologically show a peak in the active phase and a decline in the resting phase [2, 3].

AGT is produced not only in the liver but also in adipose tissue. In rodents, AGT production was shown to contribute as much as one-third of the circulating AGT level using AGT-deficient mice [12]. Obese subjects are reported to show altered circadian blood pressure rhythm, with an increase in the prevalence of the nondipper type status [13]. Thus, the present study by Matsuyama et al. also suggests that "adipose tissue-derived AGT" may be increased and play a harmful role in organ damage in obese patients when accompanied by renal dysfunction. There may be a vicious cycle in obese CKD patients who have elevated AGT production, which increases glomerular damage [14] (Fig. 1).

However, in addition to the study limitations described in the Discussion, the present study raises several 
Fig. 1 Effect of liver-derived angiotensinogen (AGT) on normal glomeruli and damaged glomeruli. L-AGT liver-derived angiotensinogen, A-AGT adipocyte-derived angiotensinogen, P-AGT proximal tube-derived angiotensinogen, RAS renin-angiotensin system

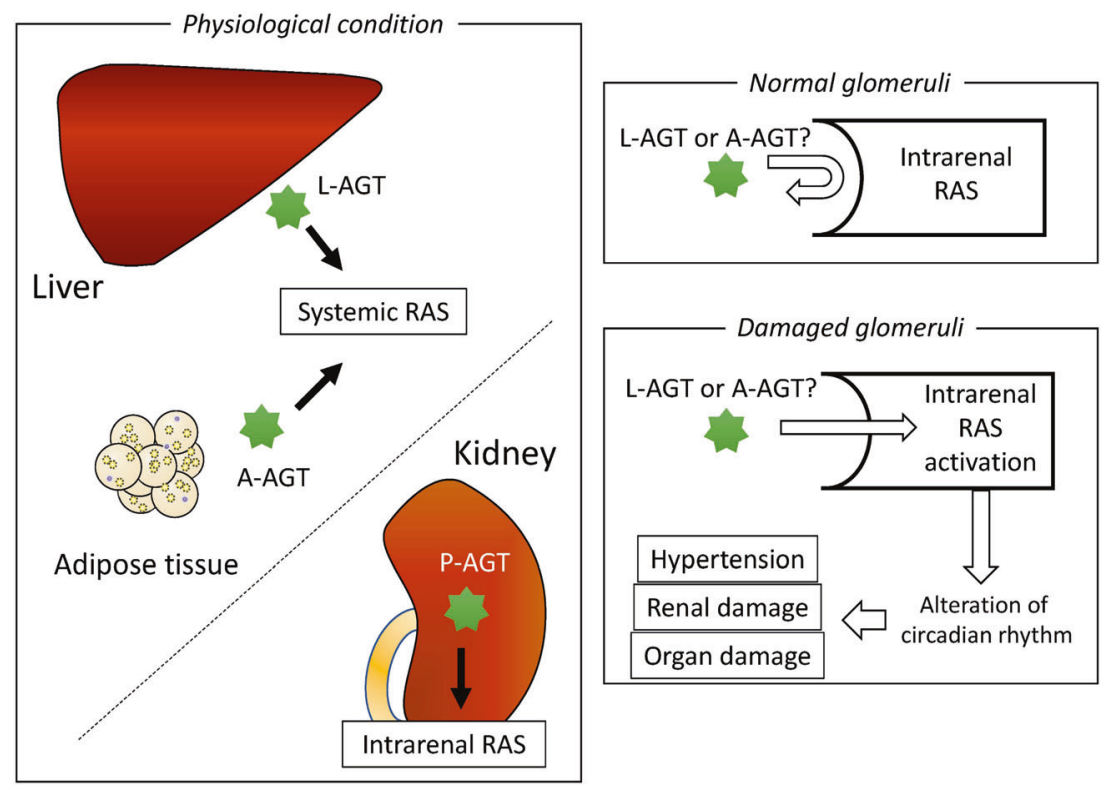

questions. First, Takenaka et al. demonstrated that plasma angiotensin II levels are increased twofold in Adriamycin nephropathy rats [15]. Thus, what are the direct effects of the filtration of circulating angiotensin II on intrarenal RAS activation? Second, is it possible to prevent the increase in intrarenal AGT in these nephropathy rats by using an inhibitor of podocyte injury, such as a mineralocorticoid receptor blocker? Third, whether filtered liverderived AGT actually activates the intrarenal RAS has not been elucidated; thus, according to the description in the Discussion, future studies using liver-specific AGT gene knockout mice are needed.

A physical barrier such as the glomerulus not only functions in the exchange of substances but also maintains the tissue microenvironment. Disturbance of the barrier induces tissue dysfunction by the accumulation of small changes in the microenvironment and leads to organ failure. Thus, the present study again indicates that attention should be given to the disturbance in circadian rhythms observed in the evaluation of circadian blood pressure rhythm and blood pressure variability. If an abnormal circadian blood pressure rhythm is recognized, disorders of glomerular capillary pressure and intrarenal RAS activation should be investigated, and appropriate treatment should be started to prevent changes in the microenvironment of the kidney.

\section{Compliance with ethical standards}

Conflict of interest The author declares no competing interest.

Publisher's note Springer Nature remains neutral with regard to jurisdictional claims in published maps and institutional affiliations.

\section{References}

1. Takeda N, Maemura K. Circadian clock and the onset of cardiovascular events. Hypertens Res. 2016;39:383-90.

2. Firsov D, Bonny O. Circadian regulation of renal function. Kidney Int. 2010;78:640-5.

3. Firsov D, Bonny O. Circadian rhythms and the kidney. Nat Rev Nephrol. 2018;14:626-35.

4. Isobe S, Ohashi N, Ishigaki S, Tsuji T, Sakao Y, Kato A, et al. Augmented circadian rhythm of the intrarenal renin-angiotensin systems in anti-thymocyte serum nephritis rats. Hypertens Res. 2016;39:312-20.

5. Isobe S, Ohashi N, Fujikura T, Tsuji T, Sakao Y, Yasuda H, et al. Disturbed circadian rhythm of the intrarenal renin-angiotensin system: relevant to nocturnal hypertension and renal damage. Clin Exp Nephrol. 2015;19:231-9.

6. Ohashi N, Isobe S, Ishigaki S, Yasuda H. Circadian rhythm of blood pressure and the renin-angiotensin system in the kidney. Hypertens Res. 2017;40:413-22.

7. Matsuyama $\mathrm{T}$, Ohashi $\mathrm{N}$, Aoki $\mathrm{T}$, Ishigaki S, Isobe $\mathrm{S}$, Sato $\mathrm{T}$, et al. Circadian rhythm of the intrarenal renin-angiotensin system is caused by glomerular filtration of liver-derived angiotensinogen depending on glomerular capillary pressure in adriamycin nephropathy rats. Hypertens Res. 2021 (e-pub ahead of print 2021/02/10; https://doi.org/10.1038/s41440-02100620-6).

8. Kobori H, Nangaku M, Navar LG, Nishiyama A. The intrarenal renin-angiotensin system: from physiology to the pathobiology of hypertension and kidney disease. Pharm Rev. 2007;59:251-87.

9. Kobori H, Nishiyama A, Harrison-Bernard LM, Navar LG. Urinary angiotensinogen as an indicator of intrarenal Angiotensin status in hypertension. Hypertension 2003;41:42-9.

10. Nishiyama A, Kobori $H$. Independent regulation of reninangiotensin-aldosterone system in the kidney. Clin Exp Nephrol. 2018;22:1231-9.

11. Matsusaka T, Niimura F, Shimizu A, Pastan I, Saito A, Kobori H, et al. Liver angiotensinogen is the primary source of renal angiotensin II. J Am Soc Nephrol. 2012;23:1181-9.

12. Massiera F, Bloch-Faure M, Ceiler D, Murakami K, Fukamizu A, Gasc JM, et al. Adipose angiotensinogen is involved in adipose 
tissue growth and blood pressure regulation. FASEB J. 2001;15: 2727-9.

13. Kotsis V, Stabouli S, Bouldin M, Low A, Toumanidis S, Zakopoulos N. Impact of obesity on 24-hour ambulatory blood pressure and hypertension. Hypertension 2005;45:602-7.

14. Kanzaki G, Tsuboi N, Haruhara K, Koike K, Ogura M, Shimizu A, et al. Factors associated with a vicious cycle involving a low nephron number, hypertension and chronic kidney disease. Hypertens Res. 2015;38:633-41.

15. Takenaka $\mathrm{T}$, Inoue $\mathrm{T}$, Miyazaki $\mathrm{T}$, Kobori H, Nishiyama A, Ishii $\mathrm{N}$, et al. Klotho suppresses the renin-angiotensin system in adriamycin nephropathy. Nephrol Dial Transpl. 2017;32: 791-800. 